\title{
Relaxation oscillations suppression and undamping in a hybrid photonic crystal laser
}

Kovalev, Anton, Butler, Sharon, Bakoz, Andrei, Hegarty, Stephen, O'Faolain, Liam, et al.

Anton V. Kovalev, Sharon M. Butler, Andrei Bakoz, Stephen Hegarty, Liam O'Faolain, Evgeny Viktorov, "Relaxation oscillations suppression and undamping in a hybrid photonic crystal laser," Proc. SPIE 11356,

Semiconductor Lasers and Laser Dynamics IX, 113560P (1 April 2020); doi: $10.1117 / 12.2556531$

SPIE. Event: SPIE Photonics Europe, 2020, Online Only 


\title{
Relaxation oscillations suppression and undamping in a hybrid photonic crystal laser
}

\author{
Anton V. Kovalev ${ }^{\mathrm{a}}$, Sharon M. Butler ${ }^{\mathrm{b}, \mathrm{c}}$, Andrei Bakoz ${ }^{\mathrm{b}, \mathrm{c}}$, Stephen Hegarty ${ }^{\mathrm{b}, \mathrm{c}}$, Liam \\ $\mathrm{O}^{\prime}$ Faolain $^{\mathrm{b}, \mathrm{c}, \mathrm{d}}$, and Evgeny Viktorov ${ }^{\mathrm{a}}$ \\ ${ }^{a}$ ITMO University, Birzhevaya Liniya 14, Saint Petersburg, Russia \\ ${ }^{\mathrm{b}}$ Centre for Advanced Photonics \& Process Analysis, Cork Institute of Technology, Cork, \\ Ireland \\ 'Tyndall National Institute, Lee Maltings, Dyke Parade, Cork, Ireland \\ ${ }^{d}$ Scottish Universities Physics Alliance (SUPA), School of Physics and Astronomy, North \\ Haugh, St. Andrews, UK
}

\begin{abstract}
We explore the short-cavity hybrid laser consisting of a III-V amplifier integrated with a silicon photonic crystal $(\mathrm{PhC})$ cavity reflector using the so-called butt-coupling approach. The laser possesses great stability characteristics meeting the criteria for data center interconnect applications. The PhC reflector having a Q-factor of $10^{4}$ at a lasing wavelength $1535 \mathrm{~nm}$ can be considered as a narrow-bandwidth filter. The laser demonstrates single mode and eventless operation without any dynamics on the background, and featureless radiofrequency spectrum without evidence of relaxation oscillation frequency. We confirm our experimental observations by theory based on a delay differential equation model for a single-section semiconductor laser. We reveal the effective damping of the laser, when the detuning between the filter peak and the laser cavity mode is small, and the imaginary parts of the model eigenvalues equal zero. Undamping of the relaxation oscillations is also possible forcing self-Q-switched operation in the laser owing to the cumulative action of the alpha-factor and the narrow filter.
\end{abstract}

Keywords: photonic crystal cavity, semiconductor laser, hybrid laser, integrated photonics, relaxation oscillations, damping

\section{INTRODUCTION}

As demand for cloud-based services and high-performance computation grows, it imposes requirements on data center performance, in particular efficiency. In turn, there is a necessity for more efficient and faster optical interconnects and optical intra-chip communications, lying in the core of the data centers. The rising field of silicon photonics provides means to meet these harsh requirements by accommodation of both electrical and optical components. Taking advantage of the mature CMOS process technology, and the fact that silicon is the basic material of electronics industry, silicon photonics makes possible photonic integrated circuits for use in highly efficient and fast communications at large volumes. As the main power consuming optical interconnect blocks are the light source and modulator, significant efforts have been made toward development of effective $\mathrm{Si}$ optical devices. Progress in effective micro-photonic resonator based components, such as ring resonators, integrated waveguides and photonic crystal cavities, made valid a chip scale integration of modulators with sub-pJ/bit switching energies. ${ }^{1}$

Here we explore a short-cavity hybrid laser consisting of a III-V amplifier integrated with a silicon photonic crystal (PhC) cavity reflector using the butt-coupling approach. ${ }^{2}$ The laser possesses good stability characteristics meeting the criteria for data center interconnect applications. The $\mathrm{PhC}$ reflector having a $\mathrm{Q}$-factor of on the order $10^{4}$ at the lasing wavelength $1535 \mathrm{~nm}$ can be considered as an intracavity narrow-bandwidth filter. The laser can demonstrate single mode and eventless operation without any dynamics on the background, and smooth

Further author information: (Send correspondence to A.V.K.)

A.V.K.: E-mail: avkovalel@niuitmo.ru

Semiconductor Lasers and Laser Dynamics IX, edited by Marc Sciamanna, Rainer Michalzik,

Krassimir Panajotov, Sven Höfling, Proceedings of SPIE Vol. 11356, 113560P

(C) 2020 SPIE · CCC code: $0277-786 X / 20 / \$ 21 \cdot$ doi: 10.1117/12.2556531

Proc. of SPIE Vol. 11356 113560P-1 
radiofrequency spectrum without evidence of relaxation oscillation frequency. The latter fact indicates extremely high damping in $\mathrm{PhC}$ laser, where the photon cavity lifetime is greatly increased by the high-Q $\mathrm{PhC}$ cavity reflector.

We confirm our experimental observations by theory based on a delay differential equation (DDE) model for a single-section semiconductor laser. We analyze the stability of the continuous wave solution, and use a first-order approximation of the eigenvalue spectrum valid for narrow filter bandwidth. In this case the nontrivial eigenvalues of the system are associated with relaxation oscillations, and effective damping of the laser is revealed when the detuning between the filter peak and the laser cavity mode is small, and the imaginary parts of eigenvalues equal zero. It is interesting though that if the detuning is large, the photon cavity lifetime decreases, and it is possible to undamp relaxation oscillations forcing self-Q-switched operation in the laser. The latter effect is caused by the combined action of the alpha-factor leading to frequency modulation, and the narrow filter converting this modulation into amplitude modulation of the field which drives the system into an off-equilibrium state.

\section{EXPERIMENTAL RESULTS}

Summing up all the definitions of the $Q$-factor, it could be considered as a quantitative indicator of the spatial mode confinement. The modal volume describes the spatial extent and the energy distribution of the mode inside the cavity. Taking into account that the $\mathrm{PhC}$ cavity is formed in a photonic band gap (meaning there is no propagation into the surrounding lattice) and has a volumetric size as small as order of $\lambda^{3}$, the values of $Q$ are very high. For this reason, a replacement of distributed gratings with $\mathrm{Si}_{3} \mathrm{~N}_{4}$ resonant mirrors, such as $1 \mathrm{D}$ and 2D photonic crystal cavities, ${ }^{3}$ whose high $Q$-factors and incredibly small footprints (even compared to gratings), would lead to a finer lasing wavelength control and wider mode-hop free regime.

We explore the hybrid laser configuartion that was described $i^{4}$ with the possibility of reflector frequency modulation by microheater induced thermal effect, but here the $\mathrm{PhC}$ reflector remains unmodulated. Instead, a second microheater (II) was employed as a phase tuning (PT) tool. The Si dispersion adapted (DA) PhC cavity was $14 \mu \mathrm{m}$ long which corresponds to $\sim 7 \mathrm{~nm}$ free spectral range (FSR). In addition to the standard lasing condition of overlap between the gain peak and a cavity mode, the preferred lasing wavelength (1535 $\mathrm{nm}$ ) was also determined by the presence of gain ripple in the reflective semiconductor optical amplifier (RSOA) spectrum (arising due non-optimized AR coating) (Fig. 1(a)). The total propagation laser cavity phase is the most straightforward parameter for the introduction of detuning. In this experiment a DC voltage in a range $0.58-1.73 \mathrm{~V}$ in steps of $0.29 \mathrm{~V}$ has been applied to a phase-tuning (PT) section and was kept at the same value until time traces containing the resulting fast dynamical processes has been recorded. Due to translation symmetry, the cavity mode structure is $2 \pi$ invariant in terms of propagation phase. A full swing of $2 \pi$ shift corresponds to $\Delta U=1.15 \mathrm{~V}$, where $\Delta U$ difference between initial and final voltage (Fig. 1(b)). The injection current of the RSOA was $100 \mathrm{~mA}$ (with threshold at $30 \mathrm{~mA}$ ). The time traces were acquired through New Focus 1544-B $12 \mathrm{GHz}$ bandwidth photoreceiver connected to DPO73304DX fast digital oscilloscope with 33 $\mathrm{GHz}$ bandwidth, meaning that the cut-off acquisition frequency was $12 \mathrm{GHz}$. The microheater bias voltage was $0.58 \mathrm{~V}(6.7 \mathrm{~mW})$ and it can be viewed as an initial voltage corresponding to $0 \pi$ shift of the total propagation phase. Similar to conventional DFB sources, PhC laser shows monotonous SM operation for the majority of the pumping current values (Fig. 2(a)) without any dynamics on the background (Fig. 2(b)). The absence of the peaks corresponding to the relaxation oscillation frequency (ROF) in the ASE RF spectrum (Fig. 2(c)) is beneficial for multiple applications and indicates extremely high damping in $\mathrm{PhC}$ laser, where $Q$-factor could be considered as a dimensionless lifetime, indicating the number of optical periods that elapse before the energy decays by $e^{-2 \pi}$ and the decay rate $\Gamma$ is defined as $\Gamma=\frac{\omega_{0}}{2 Q}$. Owing to the small size of the $\mathrm{PhC}$ cavity (in this case $14 \mu \mathrm{m}$ long) and high $Q$-factor/Volume ratio, ${ }^{5}$ the $\mathrm{Si} \mathrm{PhC}$ cavity accommodates a high energy-density mode which leads to nonlinear behavior of the reflector depending on the amount of coupled energy. Phase tuning can be effective as a fine-tuning mechanism for coupling between the lasing cavity mode and the $\mathrm{PhC}$ resonant frequency as we demonstrate below using a delay differential equation (DDE) model. 

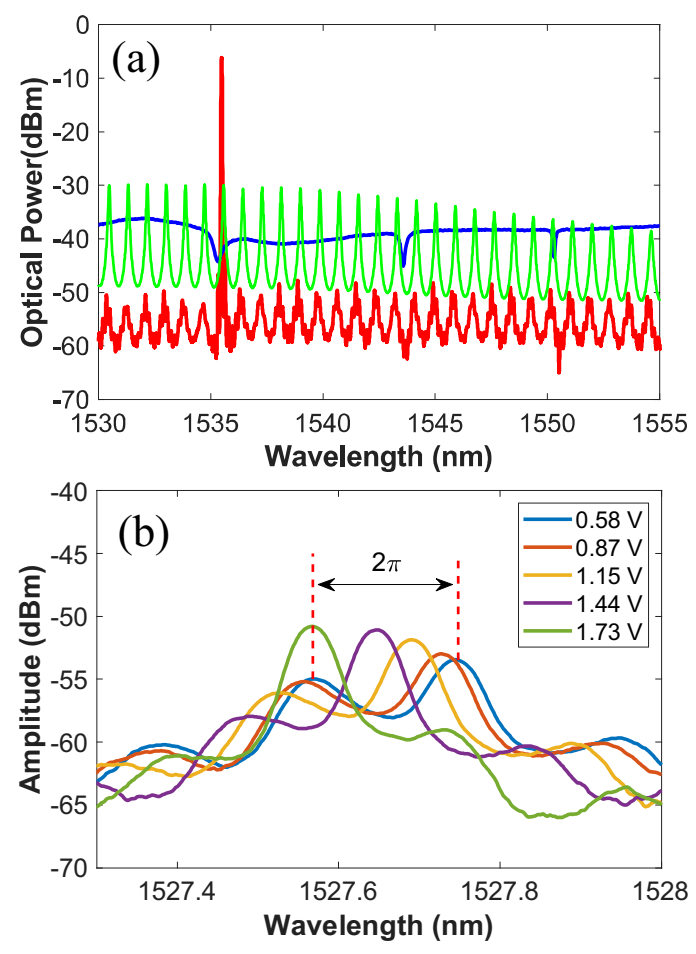

Figure 1. (a) Single mode lasing at $78 \mathrm{~mA}$ (red) at $1535 \mathrm{~nm}$ resonance peak (lasing wavelength $1535.48 \mathrm{~nm}$ ), RSOA gain spectrum (green) at $78 \mathrm{~mA}$ shown together with transmission (dark blue) spectra of the PhC cavity. (b) Optical spectrum of the $\mathrm{PhC}$ laser operated under threshold versus bias voltage applied on microheater. The fringes with $\sim 0.18 \mathrm{~nm}$ spacing correspond to the FSR of the laser cavity. $2 \pi$ propagation phase shift corresponds to $\Delta U=1.15 \mathrm{~V}(\Delta P=53.3 \mathrm{~mW})$.

\section{THEORETICAL MODEL}

We explore the DDE model, modified for a single-section semiconductor laser: ${ }^{6}$

$$
\begin{aligned}
\gamma^{-1} \frac{d E(t)}{d t}+E(t) & =\sqrt{\kappa} \exp ((1-i \alpha) G(t-\tau) / 2-i \Omega \tau) E(t-\tau), \\
\gamma_{g}^{-1} \frac{d G(t)}{d t} & =g_{0}-G(t)-\left(e^{G(t)}-1\right)|E(t)|^{2},
\end{aligned}
$$

where $E(t)$ is the complex electric field amplitude; $t$ is time; $\tau$ is the cavity round-trip time; $\gamma$ is the narrow bandwidth of the laser cavity; $\kappa<1$ is the total non-resonant round-trip intensity attenuation in the cavity; $G(t)$ is the normalized gain; $\alpha$ is the linewidth enhancement factor; $\Omega$ is the angular frequency detuning of the the gain maximum with relation to the optical frequency of the closest cavity mode; $g_{0}$ is the pump parameter; $\gamma_{g}$ is the carrier recombination rate.

The model Eqs. (1)-(2) admits rotating wave or CW solutions in the form $E(t)=A_{M} \exp i \omega_{M} t, G(t)=G$ which can be written parametrically as:

$$
\begin{aligned}
\Omega & =-\frac{\alpha}{2 \tau} \ln \left(\frac{\gamma^{2}+\omega_{M}^{2}}{\gamma^{2} \kappa}\right)-\omega_{M}+2 \tau^{-1} \tan ^{-1}\left(\frac{\gamma-\sqrt{\gamma^{2}+\omega_{M}^{2}}}{\omega_{M}}\right)+2 \pi M \tau^{-1} \\
A_{M} & =\sqrt{\frac{g_{0}-G}{e^{G}-1}} \\
G & =\ln \left(\frac{\gamma^{2}+\omega_{M}^{2}}{\gamma^{2} \kappa}\right)
\end{aligned}
$$



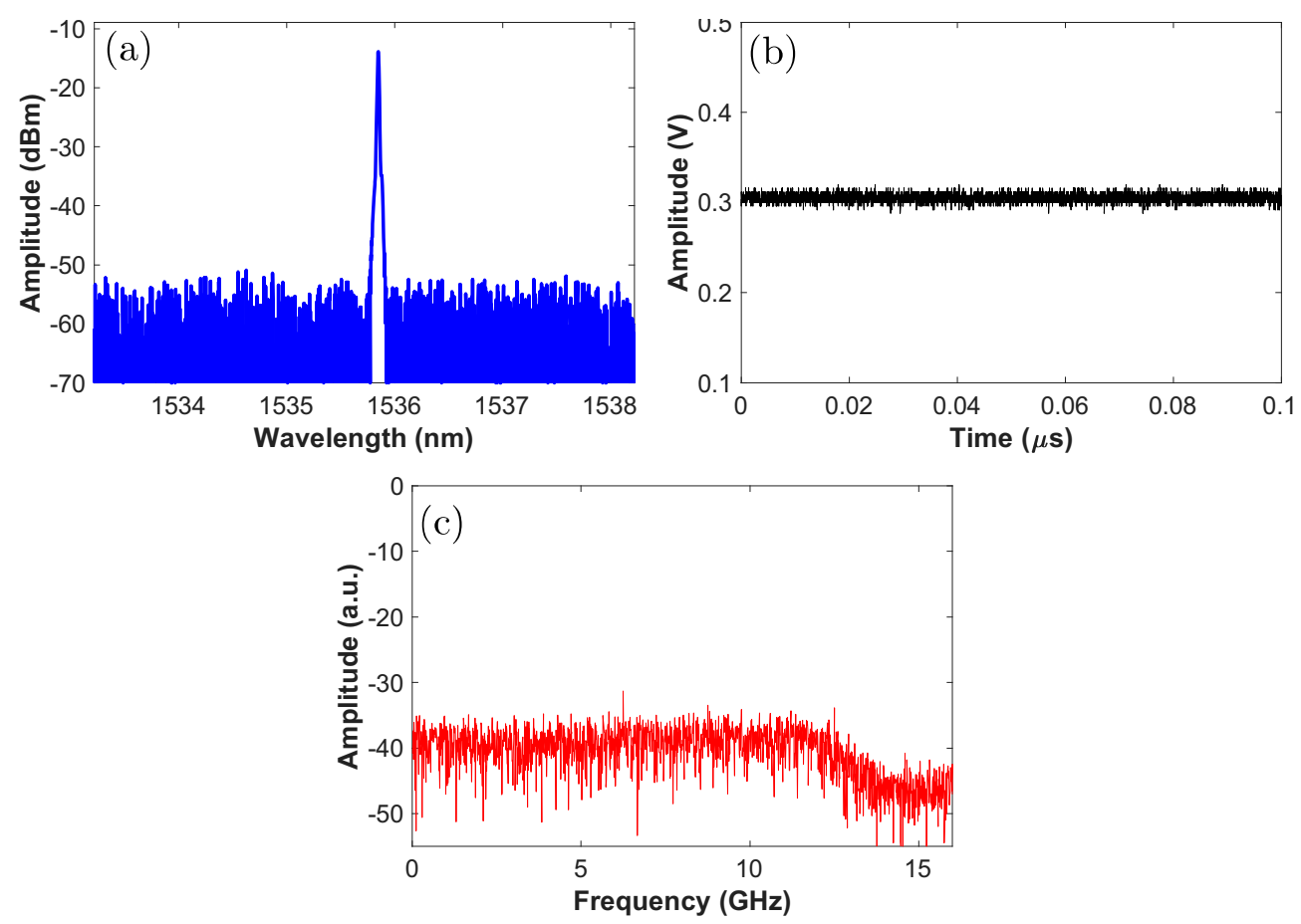

Figure 2. (a) Optical spectrum single mode regime at single resonance (1535 nm). (b) Time trace of (a) without any dynamics. (c) Fourier spectrum of (b) shows that this is indeed eventless regime. The roll-off in the laser intensity noise at $12 \mathrm{GHz}$ is the measurement bandwidth of the experiment.

where $A_{M}$ is the CW field amplitude, $\omega_{M}$ is the frequency shift with relation to the filter peak frequency, $M$ is an integer number corresponding to the number of the $\mathrm{CW}$ solution branch.

The pump threshold $g_{0, t h r}$ is defined as:

$$
g_{0, t h r}\left(\omega_{M}\right)=\ln \frac{\omega_{M}^{2}+\gamma^{2}}{\gamma^{2} \kappa^{2}}
$$

Note that the threshold depends on the frequency shift $\omega_{M}$ which in turn depends on the detuning parameter $\Omega$. In the case of maximal gain $\left(\omega_{M}=0\right)$ the threshold is defined strictly by the linear cavity losses $\kappa$ :

$$
g_{0,0}=g_{0, t h r}(0)=-\ln \kappa
$$

Each CW branch has two fold bifurcation points $\omega_{L P_{ \pm}}$corresponding to the extrema of the function $\Omega\left(\omega_{M}\right)$ given by Eq. (3): ${ }^{7}$

$$
\omega_{L P_{ \pm}}=\frac{-\alpha \pm \sqrt{\alpha^{2}-4 \gamma \tau(\gamma \tau+1)}}{2 \tau}
$$

which appear iff the filter is narrow enough. The condition $\alpha^{2}>4 \gamma \tau(\gamma \tau+1)$ defines foldover of the branch. The solutions are unstable between the limit points $\left(\omega_{L P_{-}}<\omega_{M}<\omega_{L P_{+}}\right)$and the other solutions can be either stable or unstable. Note, that the threshold condition for the steady state branch having frequencies $\omega_{M} \geq \omega_{L P_{+}}$ may not be satisfied, and in this case the steady state branch will only have a single fold bifurcation point.

Linearizing Eqs. (1)-(2) around cw solutions Eqs. (3)-(5) yields a characteristic equation:

$$
c_{0}(\lambda)+c_{1}(\lambda) e^{-\lambda \tau}+c_{2}(\lambda) e^{-2 \lambda \tau}=0
$$


where the coefficients $c_{i}(\lambda), i=0,1,2$ are defined by:

$$
\begin{aligned}
c_{0}(\lambda)= & \left((\gamma+\lambda)^{2}+\omega_{M}^{2}\right)\left(\left(A_{M}^{2} e^{G}+1\right) \gamma_{g}+\lambda\right), \\
c_{1}(\lambda)= & -\gamma_{g}\left(\alpha A_{M}^{2} \lambda \omega_{M}+\left(A_{M}^{2}+2\right) \gamma(\gamma+\lambda)+\left(A_{M}^{2}+2\right) \omega_{M}^{2}\right) \\
& -e^{G} A_{M}^{2} \gamma_{g}\left(-\alpha \lambda \omega_{M}+\gamma(\gamma+\lambda)+\omega_{M}^{2}\right)-2 \lambda\left(\gamma(\gamma+\lambda)+\omega_{M}^{2}\right), \\
c_{2}(\lambda)= & \left(\gamma^{2}+\omega_{M}^{2}\right)\left(\left(A_{M}^{2}+1\right) \gamma_{g}+\lambda\right) .
\end{aligned}
$$

Let us consider the non-trivial solutions $(\lambda \neq 0)$ of Eq. (9). For the narrow-band filtering $\gamma \tau \ll 1$, we suppose $\lambda \tau \ll 1$, and expand the exponential terms in Eq. (9) as:

$$
\begin{aligned}
e^{-\lambda \tau} & =1-\lambda \tau+\mathcal{O}\left((\lambda \tau)^{2}\right), \\
e^{-2 \lambda \tau} & =1-2 \lambda \tau+\mathcal{O}\left((\lambda \tau)^{2}\right) .
\end{aligned}
$$

This then gives two non-trivial solutions of Eq. (9) that are associated with relaxation oscillations: ${ }^{8}$

$$
\lambda_{ \pm}=-\gamma_{R O} \pm i \omega_{R O}
$$

where $\omega_{R O}$ is the relaxation oscillation frequency, conventionally defined as:

$$
\omega_{R O}=\sqrt{\varepsilon I^{2}-\gamma_{R O}^{2}}
$$

where $I=A_{M}^{2}$ is the laser field intensity, $\varepsilon$ is an analogue to the ratio of the cavity photon and carrier lifetimes, defined as:

$$
\varepsilon=\frac{\gamma_{g}\left(\gamma^{2}(1-\kappa)+\omega_{M}^{2}\right)\left(\omega_{M}\left(\alpha+\tau \omega_{M}\right)+\gamma^{2} \tau+\gamma\right)}{\gamma^{2} \kappa(2 \gamma \tau+1)}
$$

and $\gamma_{R O}$ corresponds to the relaxation oscillation damping:

$$
\gamma_{R O}=\frac{\gamma_{g}}{2}\left(\frac{I\left(\alpha \gamma^{2}(\kappa-1) \tau \omega_{M}-\alpha \tau \omega_{M}^{3}+\gamma^{3}(\kappa+1) \tau+\gamma^{2}+\omega_{M}^{2}(\gamma \tau+1)\right)}{\gamma^{2} \kappa(2 \gamma \tau+1)}+1\right)
$$

The relaxation oscillation frequency $\omega_{R O}$ vanishes for $\gamma_{R O}^{2}>\varepsilon A_{M}^{2}$, meaning effective damping. Contrariwise, the relaxation oscillations become undamped when $\gamma_{R O}<0$.

Let us analyze the case of a semiconductor laser with the following parameters: the cavity round trip time is $\tau=50 \mathrm{ps}$, the carrier relaxation time $\gamma_{g}^{-1}=1 \mathrm{~ns}$, and the linewidth enhancement factor $\alpha=1$. We plot the dependence of $\gamma_{R O}$ and $\omega_{R O}$ on the frequency detuning parameter $\Omega$ and the filter width $\gamma$ for a fixed attenuation parameter value $\kappa=0.1$ (Fig. 3(a,b)), on the detuning parameter $\Omega$ and the attenuation parameter $\kappa$ for fixed filter width value $\gamma=1 \mathrm{GHz}$ (Fig. 3(c,d)). In all cases we take only the $\mathrm{CW}$ branch part with frequencies lying before the fold point $\left(\omega>\omega_{L P_{+}}\right)$, and pump parameter was four times the threshold value for maximal gain $g_{0}=4 g_{0,0}$.

It is clearly seen that when the system is approaching the fold bifurcation by increasing the detuning $\Omega$ the relaxation oscillation frequency vanishes, and the laser becomes effectively damped under realistic parameter region denoted by the letter $D$ in Fig. 3. Additionally, when the losses in the system increase ( $\kappa$ decrease) or the width of the filter $\gamma$ increases it is possible to undamp the relaxation oscillations leading to the self-pulsing regime at the relaxation oscillation frequency within the parameter region denoted by the letter $U$ in Fig. 3 .

\section{CONCLUSION}

We experimentally and theoretically demonstrated that relaxation oscillations can be suppressed in a shortcavity semiconductor laser with a narrow intracavity frequency filter. Additionally, on the basis of our analysis we expect the undamping of relaxation oscillations, and self-pulsations when the cavity mode is detuned from the filter peak frequency. The results might be useful for applications in data communications. 

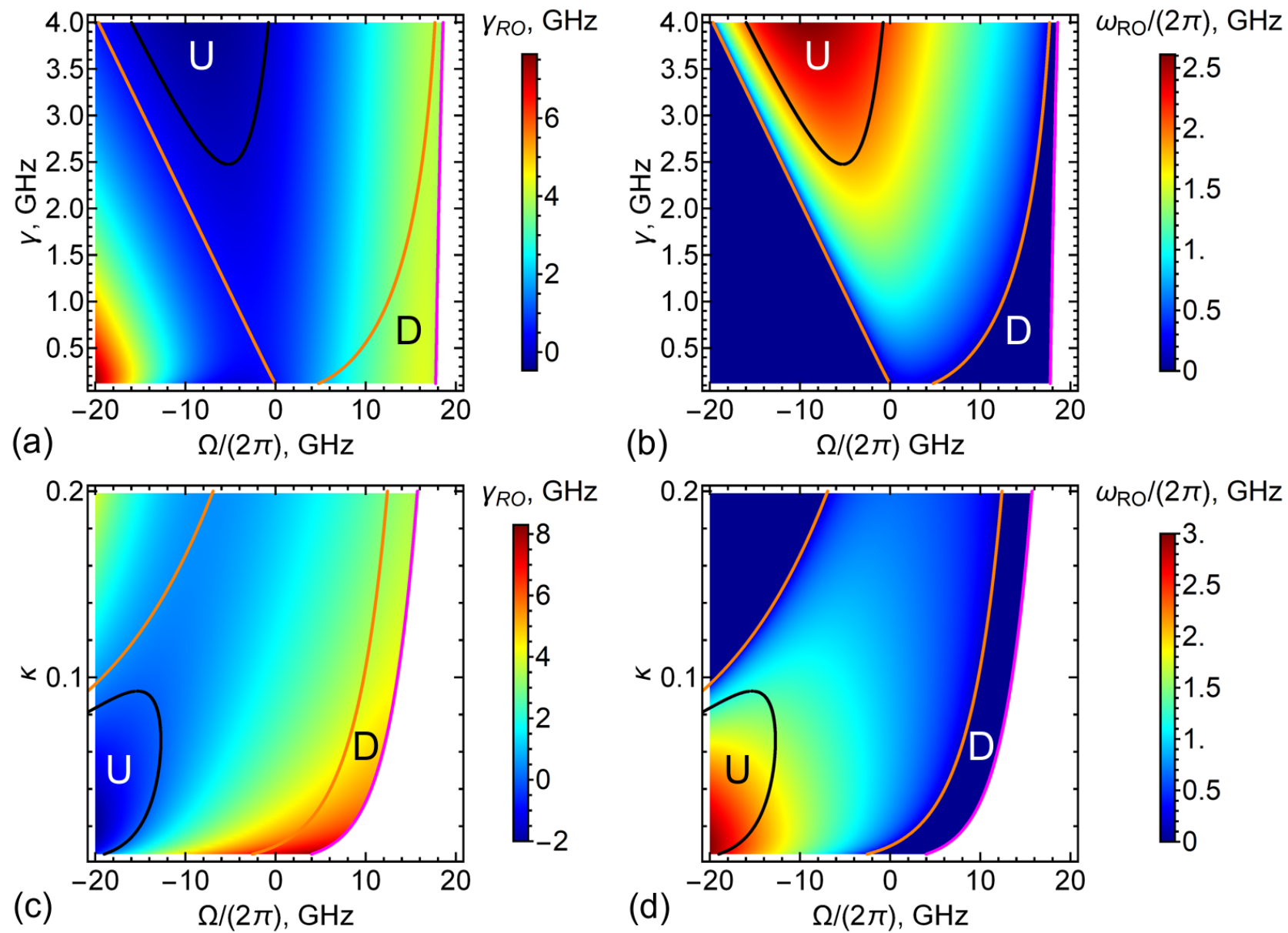

$\omega_{\mathrm{RO}} /(2 \pi), \mathrm{GHz}$

Figure 3. The dependence of the relaxation oscillation damping rate $\gamma_{R O}(\mathrm{a}, \mathrm{c})$ and frequency $\omega_{R O}(\mathrm{~b}, \mathrm{~d})$ on the frequency detuning parameter $\Omega$ and filter width $\gamma$ for the fixed attenuation parameter value $\kappa=0.1$ (a, b), on the detuning parameter $\Omega \tau$ and the attenuation parameter $\kappa$ for fixed filter width value $\gamma=1 \mathrm{GHz}(\mathrm{c}, \mathrm{d})$. The magenta lines denote the fold bifurcation border defined by $\omega_{L P_{+}}$(see Eq. 8). The black lines correspond to the border of the relaxation oscillation undamping when $\gamma_{R O}$ becomes negative. The orange lines correspond to the vanishing of relaxation oscillation frequency when $\omega_{R O}$ becomes zero, and effective damping of oscillations. The corresponding region, where the relaxation oscillations are undamped (damped) are marked by the letter $U(D)$. Only the $\mathrm{cw}$ branch parts with frequencies lying before the fold point $\left(\omega>\omega_{L P_{+}}\right)$are shown. The other parameters are: $\tau=50 \mathrm{ps}, \gamma_{g}^{-1}=1 \mathrm{~ns}, g_{0}=4 g_{0,0}$, and $\alpha=1$.

\section{ACKNOWLEDGMENTS}

Science Foundation Ireland (SFI 12/RC/2276, SFI 16/ERCS/3838 ); H2020 European Research Council (ERC) (starting grant 337508); Cork Institute of Technology (CIT) (Rísam); Irish Government's Programme for Research in Third Level Institutions, Cycle 5, Strand 1a (CREATE), H2020 PICCOLO Project - Multimodal highly-sensitive photonics endoscope for improved in-vivo colon cancer diagnosis and clinical decision support (https://www.piccolo-project.eu/). A.V.K. and E.A.V. work was supported by the Ministry of Science and Higher Education of Russian Federation, research project no. 2019-1442.

\section{REFERENCES}

[1] Zheng, X., Lin, S., Luo, Y., Yao, J., Li, G., Djordjevic, S. S., Lee, J.-H., Thacker, H. D., Shubin, I., Raj, K., Cunningham, J. E., and Krishnamoorthy, A. V., "Efficient WDM laser sources towards terabyte/s silicon photonic interconnects," Journal of Lightwave Technology 31, 4142-4154 (dec 2013). 
[2] Bakoz, A. P., Liles, A. A., Gonzalez-Fernandez, A. A., Habruseva, T., Hu, C., Viktorov, E. A., Hegarty, S. P., and O'Faolain, L., "Wavelength stability in a hybrid photonic crystal laser through controlled nonlinear absorptive heating in the reflector," Light: Science \& Applications 7, 39 (jul 2018).

[3] Debnath, K., O'Faolain, L., Gardes, F. Y., Steffan, A. G., Reed, G. T., and Krauss, T. F., "Cascaded modulator architecture for WDM applications," Optics Express 20, 27420 (nov 2012).

[4] Butler, S. M., Bakoz, A. P., Singaravelu, P. K. J., Liles, A. A., O’Shaughnessy, B., Viktorov, E. A., O'Faolain, L., and Hegarty, S. P., "Frequency modulated hybrid photonic crystal laser by thermal tuning," Optics Express 27, 11312 (apr 2019).

[5] Notomi, M., Nozaki, K., Shinya, A., Matsuo, S., and Kuramochi, E., "Toward fJ/bit optical communication in a chip," Optics Communications 314, 3-17 (mar 2014).

[6] Vladimirov, A. G. and Turaev, D., "Model for passive mode locking in semiconductor lasers," Physical Review A 72, 033808 (sep 2005).

[7] Kovalev, A. V., Dmitriev, P. S., Vladimirov, A. G., Pimenov, A., Huyet, G., and Viktorov, E. A., "Bifurcation structure of a swept-source laser," Physical Review E 101, 012212 (jan 2020).

[8] Erneux, T. and Glorieux, P., [Laser Dynamics], Cambridge University Press, Cambridge (2010). 